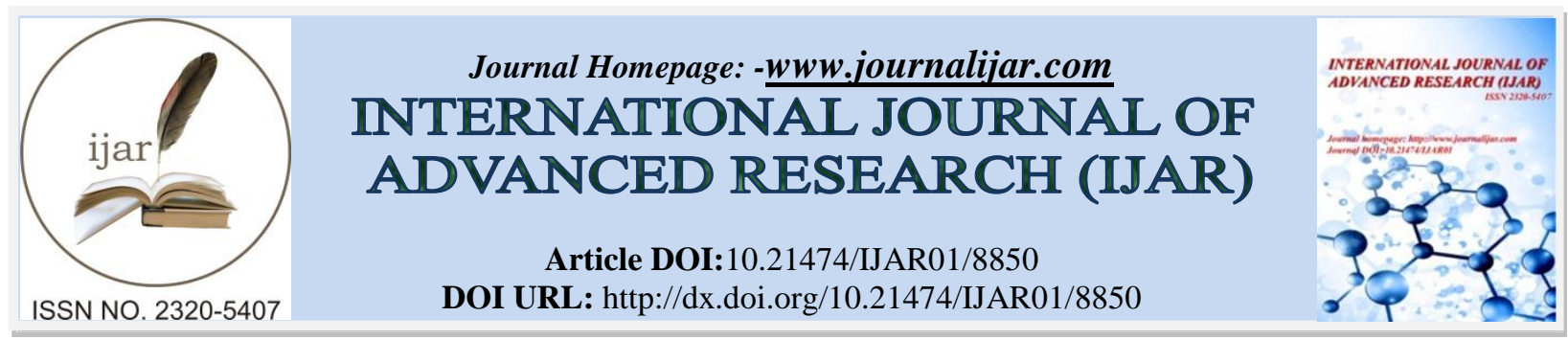

RESEARCH ARTICLE

\title{
A COMPARATIVE EVALUATION OF THE ANTIBACTERIAL AND ANTIFUNGAL EFFICACY OF DIFFERENT ROOT-END FILLING MATERIALS USING TUBE DILUTION METHOD - AN IN VITRO STUDY.
}

\section{Dr. Unnati D. Desai, Dr. Vijay Ugale, Dr. Trupti Killeker, Dr. Meenal Gulve, Dr. Swapnil Kolhe and Dr. Gayatri Aher.}

\section{Manuscript Info}

Manuscript History

Received: 08 February 2019

Final Accepted: 10 March 2019

Published: April 2019

\section{Abstract}

\section{Introduction:-}

Microorganisms are considered to be the primary etiological agents in the development and progression of pulpal and periapical diseases as well as in endodontic treatment failure. When this healing is not achieved after nonsurgical endodontic therapy and when the retreatment is not possible or failed, surgical approach is required to conserve the tooth. This procedure involves exposure of the involved apex, root resection, preparation of a class I cavity at the resected root end and insertion of a root end filling material in the prepared cavity. The root end filling material should provide an apical seal at the end of the resected root, preventing the movement of the bacteria and the bacterial products from the root canal system to the periapical tissues. ${ }^{1}$

An ideal root end filling material should produce a complete apical seal, be non toxic, biocompatible, nonresorbable, dimensionally stable, easy to manipulate, radio-opaque and well tolerated by the periapical tissues. In addition to these, the root end filling materials should have some antibacterial and antifungal activity to prevent the bacterial and fungal growth.

Retrograde filling materials are used with the purpose of obtaining root end sealing in the endodontic surgeries. In addition to improving the sealing of the existing root canal filling, these materials should possess antimicrobial properties to prevent the movement of bacteria and their products from the root canal system to the periapical tissues for the success of the endodontic surgeries. ${ }^{2}$

Mineral trioxide aggregate is marketed as gray and white preparations both of which are composed of $75 \%$ Portland cement clinker, 20\% Bismuth oxide and 5\% gypsum by weight. MTA consists of fine hydrophilic particles that, in the presence of water or moisture forms colloidal gel that solidifies to form hard cement within approximately 4 hours. The white colour preparation lacks tetra calcium aluminoferrite as a result of which, it is more aesthetic. Biodentine is a calcium based cement recently introduced new endodontic material. The powder mainly consists of tricalcium and dicalcium silicate as well as calcium carbonate. The liquid consists of calcium chloride in aqueous solution with a mixture of polycarboxylate which sets in 12 minutes. Biodentine is a bioactive dentine substitute based on Active Biosilicate Technology. Other properties are similar to MTA but setting time and handling properties are better than MTA. ${ }^{3}$

Most common predominant micro-organisms in persistent or refractory periapical lesions are facultative bacteria and yeast - E. faecalis and Candida albicans. Hence these two micro-organisms were selected for this study. 
The purpose of this study was to investigate and compare the antibacterial and antifungal effects of MTA, Biodentine and White MTA+2\% Chlorhexidine on Enterococcus faecalis and Candida albicans microorganisms.

\section{Materials And Methods:-}

1. Three root-end materials were tested for antibacterial activity against Enterococcus faecalis and antifungal activity against Candida albicans.

2. Materials used in the study were MTA Angelus (White MTA), Safe Plus (2\% Chlorhexidine) and Biodentine as shown in Fig 1.

3. The materials evaluated for antimicrobial and antifungal efficacy were divided into

4. GROUP I - White MTA

5. GROUP II - White MTA $+2 \%$ Chlorhexidin

6. GROUP III - Biodentine

7. 9 test-tubes were arranged in a row.

8. $200 \mathrm{mg}$ of each material was dissolved in $20 \mathrm{ml}$ of Sterile Brain Heart Infusion (BHI) broth. This solution was called the master dilution and contains $10 \mathrm{mg}$ of material per $1 \mathrm{ml}$ of broth.

9. Further dilutions were prepared from tube no. 2 to 6 such that subsequent test tubes had half the concentration than the previous.

10. 10 microliter of Enterococcus faecalis was added to tube no. 1-6 and 8 .

11. Tube no. 7 was used to check for sterility of material.

12. Tube no. 8 and 9 were used as positive and negative control respectively.

13. All test-tubes were incubated at $35^{\circ} \mathrm{C}$. Subcultures from each tube were made on Mac Conkey's agar and read at end of 24 hours of incubation. (Fig 2)

14. For antifungal efficacy, similar methodology was followed.Sabouraud's dextrose broth (SDB) and Sabouraud's dextrose agar (SDA) were used for dilutions and cultures (Fig. 2). Strains of Candida albicans were used for evaluation of antifungal efficacy of the materials.

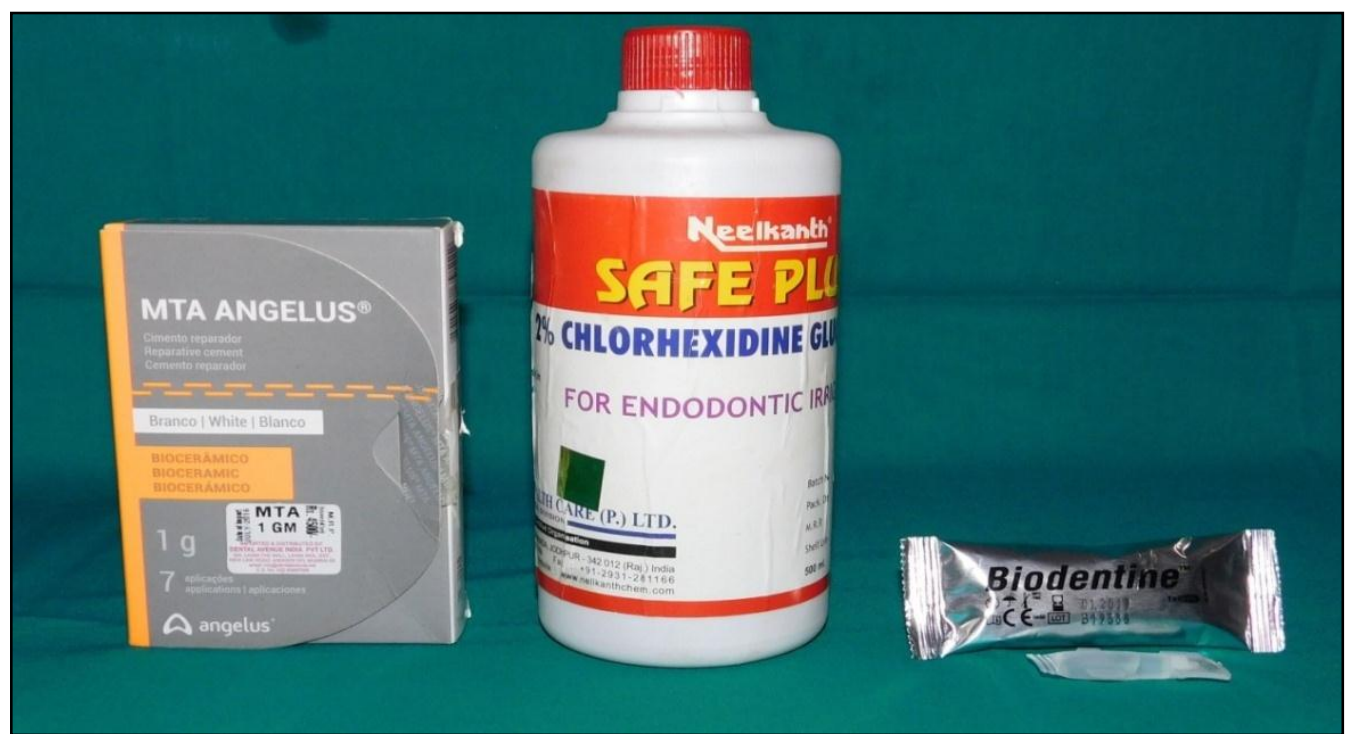

Fig1:-Materials used in the the study 


\section{Subculture on MacConkey's agar for Group I, II,III}

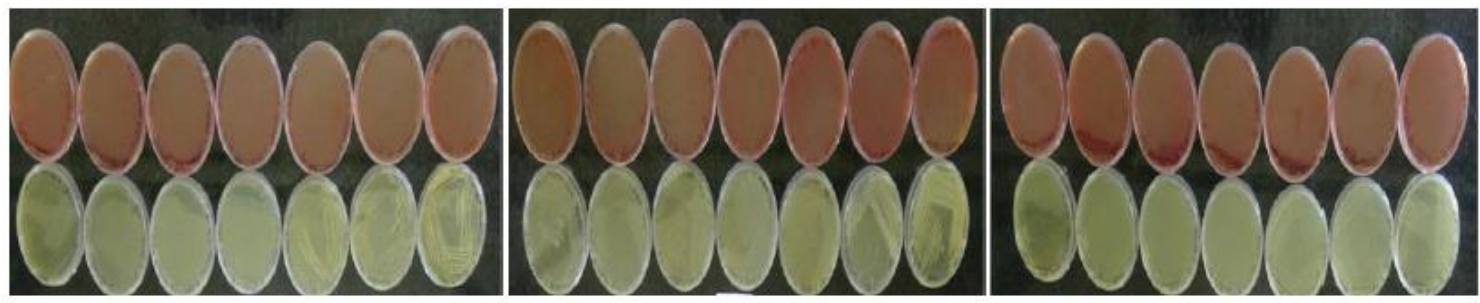

Subculture on Sabouraud's dextrose agar for Group I, II, III

\section{Result:-}

Fig 2:-Subculture of Enterococcus faecalis and Candida albicans

The results were tabulated and statistically analyzed using one-way analysis of variance (ANOVA) and MannWhitney Test using Statistical Package for Social Sciences (SPSS).

The antibacterial efficacy was found to be best for Group III ( Biodentine) followed by Group II (White MTA + Chlorhexidine) (Table 1).The antifungal activity was similar for both Group II (White MTA + Chlorhexidine) and Group III (Biodentine)(Table 2).While Group I (White MTA) showed least antibacterial and antifungal efficacy as compared to the other two groups. There was a statistically significant difference between antibacterial efficacy among all groups and antifungal efficacy between White MTA + CHX and Biodentine was not significant (Table $3)$.

Table 1:-Minimum inhibitory concentration and mean value for Enterococcus faecalis

\begin{tabular}{|c|c|c|c|}
\hline Groups & Minimum & Maximum & Mean \\
\hline White MTA & 0.0 & 1.25 & 0.625 \\
\hline White MTA + CHX & 0.0 & 0.625 & 0.312 \\
\hline Biodentine & 0.0 & 0.3 & 0.156 \\
\hline
\end{tabular}

Table 2:-Minimum inhibitory concentration and mean value for Candida albicans

\begin{tabular}{|c|c|c|c|}
\hline Groups & Minimum & Maximum & Mean \\
\hline White MTA & 0.0 & No inhibition & - \\
\hline White MTA + CHX & 0.0 & 1.25 & 0.625 \\
\hline Biodentine & 0.0 & 1.25 & 0.625 \\
\hline
\end{tabular}

Table 3:-Group wise comparision by Mann-Whitney Test

\begin{tabular}{|c|c|c|}
\hline Groups compared & p -value for Enterococcus faecalis & $\mathbf{p}$ - value for Candida albicans \\
\hline White MTA vs White MTA+CHX & $<0.05$ (Significant) & $<0.05$ (Significant) \\
\hline White MTA vs Biodentine & $<0.05$ (Significant) & $<0.05$ (Significant) \\
\hline White MTA+CHX vs Biodentine & $<0.05$ (Significant) & $>0.05$ (Not Significant) \\
\hline
\end{tabular}


Graph 1:-Minimum inhibitory concentration for Enterococcus faecalis and Candida albicans

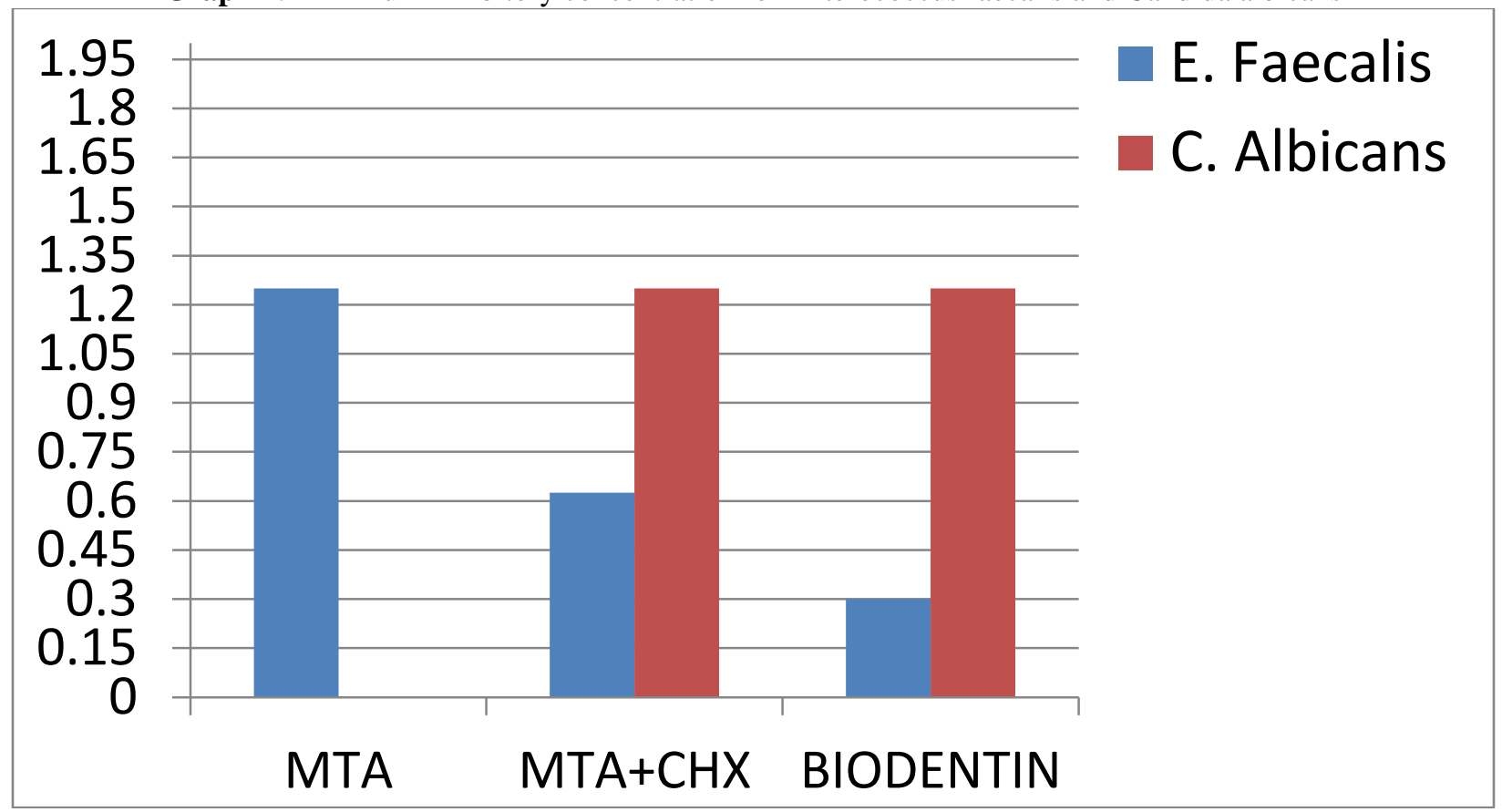

\section{Discussion:-}

The success of the root canal treatment depends on careful diagnosis and accurately performed cleaning, shaping and compact filling of the root canal under aseptic conditions. Most pulpal and periapical disease are best managed nonsurgically. However, certain microorganisms are repeatedly recovered from previously root-filled teeth that have become infected. ${ }^{1}$ In the presence of pathological lesion that cannot be eliminated by endodontic treatment or in case root canal retreatment cannot be performed or retreatment fails, endodontic surgery procedures are applied. ${ }^{2}$ Following apical resection, retrograde filling is performed in order to prevent the transmission of tissue fluid into the canal or the transmission of the microorganisms or the toxins inside the canal into the periapical tissues in the teeth in which root canal filling is performed. To this end, a lot of materials called the root-end filling materials having different physical, chemical and biological properties are used. ${ }^{3}$ An ideal root-end filling material should have following ideal requirements-

1. The most important requirement of a root endmaterial is that it should be biocompatibleand non-toxic, as it placed in direct contactwith vital soft tissue.

2. It should provide a biological seal. i.e. should promote cementum deposition on the cut root surface.

3. It should adhere to tooth structure.

4. It should be insoluble in tissue fluids.

5. It should be dimensionally stable.

6. It should be non resorbable.

7. It should be radio opaque. ${ }^{4}$

Root-end filling materials cannot provide perfect sealing and thus, there remain microscopic spaces between the retrograde cavity and the filling material. ${ }^{5}$ Microorganisms and their products may penetrate into root canal system through these spaces. Therefore, antimicrobial activities of the materials used as root-end filling material are necessary. ${ }^{6}$ MTA is one of the main retrograde filling materials frequently used today. Several studies have shown that MTA is a bioactive and biocompatible material. ${ }^{7,8}$ However, the disadvantages of MTA- its difficult manipulation and long setting time have led to development of new calcium silicate based materials. ${ }^{9}$ Biodentine is one such material. It is a tricalcium silicate based material, which is developed as a dentin restorative material and can be used in the endodontic indications of MTA. ${ }^{10}$ Hence in this study the antimicrobial efficacy of MTA, MTA+Chlorhexidine and Biodentine was compared.

The strains evaluated in this study were E. faecalis and C. albicans, which are frequently isolated during routine endodontic treatment of an infected root or from teeth with periapical pathology. They have been found with higher 
frequency in cases where treatment had been protracted, in flare-ups and in failing cases. These micro-organisms can enter the root canal system before or after treatment and may then cause secondary infections. E.faecalis was chosen as the test organism because it is associated with persistent apical inflammation in clinical situations. It has been used extensively in endodontic research because it has been found in infected canal and has been associated with failed root canal treatment. ${ }^{11}$ Fungi such as $\mathrm{C}$. albicans may gain access to the root canal from the oral cavity as a result of poor asepsis during endodontic procedures, or because of coronal leakage. C.albicans has the ability to form biofilms on different surfaces and may be involved in cases of persistent and secondary infection. ${ }^{12}$

The most common method for assessing the antimicrobial activity of root-end filling materials is agar diffusion test. But this method has disadvantages like lack of standardization of inoculums density, adequate culture medium, agar viscosity etc. In this study, tube dilution method was used to evaluate the antimicrobial efficacy of the materials. It is an efficient method for evaluating as there is a direct contact between the microorganisms and experimental materials which allows a more realistic interaction. Various dilutions of the material are prepared and the concentration at which the material was able to inhibit the growth of microorganism was recorded as the minimal inhibitory concentration (MIC) of the material. ${ }^{13}$

The results obtained were as follows- the antibacterial efficacy was found to be best for Group III ( Biodentine) followed by Group II (MTA + Chlorhexidine). The antifungal activity was similar for both Group II and Group III. While Group I (MTA) showed least antibacterial and antifungal efficacy as compared to the other two groups.

MTA fulfills major criteria's and is considered as an ideal material for root repair and root end filling. MTA has its advantages like regenerative and osteogenic potential, good marginal sealing ability, biocompatibility and bioactivity. Antibacterial properties of this calcium silicate material can be attributed to its alkalinity. The $\mathrm{pH}$ value of the freshly mixed MTA was 10.2, which increased upto 12.5 after 3 hours. This increase in the $\mathrm{pH}$ value might be due to calcium hydroxide released during the hydration process. The released hydroxyl ions as a result of ionization caused irreversible inactivation of the cellular enzymes of microganisms. ${ }^{14}$

Addition of Chlorhexidine (CHX) to MTA (Group II) resulted in better antimicrobial activity as compared to MTA (Group I).This was because of the antimicrobial properties of chlorhexidine.Chlorhexidine is a positively charged hydrophobic molecule which interacts with negatively charged phospholipids and lipopolysaccharides on the cell membrane of bacteria. This makes the cell wall permeable. The chlorhexidine molecules entered the bacteria, thus resulting in cell death. ${ }^{15}$

Biodentine (Group III) showed the highest antimicrobial activity as compared to other two groups. Biodentine is available in the form of powder and liquid. The main component of powder is a tricalcium silicate, with the addition to the powder of Calcium carbonate $(\mathrm{CaCO} 3)$ and Zirconium oxide $(\mathrm{ZrO} 2)$.The liquid is a solution of Calcium chloride $(\mathrm{CaCl} 2)$ with a water reducing agent. On setting it forms a gel structure which results in the ionic exchange. Compared to other calcium based cements, it presents a faster setting time of about 12 minutes and higher mechanical properties. Thus it is mechanically stronger, less soluble, gives a tighter seal preventing microleakages. Antimicrobial efficacy of Biodentine is attributed to the hydration of tricalcium silicate resulting in formation of colloidal gel and release of calcium hydroxide, which provides the ability to inhibit micro-organisms. ${ }^{16}$ Higher release of calcium and hence higher alkalinizing capacity as compared to MTA can be attributed to the presence of calcium silicate and calcium chloride. ${ }^{13}$

\section{Conclusion:-}

Within the limitations of the study, all the materials proved to have antimicrobial efficacy. This study showed that Biodentine had better antimicrobial efficacy as compared to the other two. And it was seen that White MTA showed better efficacy when combined with chlorhexidine owing to its cumulative benefits. 


\section{References:-}

1. Hasan ZM, Javidi M, Naderinasab M, Gharechahi M. Comparative evaluation of antimicrobial activity of three cements: New endodontic cement (NEC), mineral trioxide aggregate (MTA) and Portland. J Oral Sci 2009;51:437-42.

2. Friedman S. Retrograde approaches in endodontic therapy. Dent Traumatol 1991;7:97-107.

3. Torabinejad M, Rastegar AF, Kettering JD, Ford TRP. Bacterial leakage of mineral trioxide aggregate as a rootend filling material. J Endod 1995;21:109-12.

4. Gartner AH, Doran SO. Advances in endodontic surgery. Dent Clin N Am 1992;36:357-79.

5. Torabinejad M, Smith PW, Kettering JD, Ford TRP. Comparative investigation of marginal adaptation of mineral trioxide aggregate and other commonly used root-end filling materials. J Endod 1995;21:295-9.

6. Torabinejad M, Hong C, Ford TP, Kettering J. Antibacterial effects of some root end filling materials. J Endod 1995;21:403-6.

7. Moretton TR, Brown CE, Legan JJ, Kafrawy A. Tissue reactions after subcutaneous and intraosseous implantation of mineral trioxide aggregate and ethoxybenzoic acid cement. J Biomed Mater Res 2000;52:528-33.

8. Parirokh M, Torabinejad M. Mineral trioxide aggregate: a comprehensive literature review-part I: chemical, physical, and antibacterial properties. J Endod 2010;36:16-27.

9. Dammaschke T, Gerth HU, Züchner H, Schäfer E. Chemical and physical surface and bulk material characterization of white ProRoot MTA and two Portland cements. Dent Mater 2005;21:731-8.

10. Demiryurek EO, Ozyurek T, Gulhan T, Keskin C. Evaluation of antibacterial and antifungal activity of calcium silicate based retrograde filling materials. Int J Appl Dent Sci 2016;2:85-8.

11. Heling I, Chandler NP. Antimicrobial effect of irrigant combinations within dentinal tubules. Int Endod J 1998;31:8-14.

12. Siquiera JF, Sen BH. Fungi in endodontic infections. Oral Surg Oral Med Oral Pathol Oral Radiol Endod 2004;97:632-41.

13. Hiremath GS, Kulkarni RD, Naik BD. Evaluation of minimal inhibitory concentration of two new materials using tube dilution method: An in vitro study. J Conserv Dent 2015;18:159-62.

14. Fridland M, Rosado R. MTA solubility: a long term study. J Endod 2005;31:376-9.

15. Kandaswamy D, Venkateshbabu N. Root canal irrigants. J Conserv Dent 2010;13:256-64.

16. Kharabe V, Baliga S, Thosar N, Gaikwad U. Antimicrobial efficacy of biodentine against various microorganisms-An In vitro study. Int J Dent Res 2014;4:33-43. 